\title{
Boysenberry Polyphenols Suppressed Elevation of Plasma Triglyceride Levels in Rats
}

\author{
Shigeru MineO $^{1}$, Akane Noguchi ${ }^{1}$, Yuta NAGAKURA ${ }^{1}$, Kinji KoBori ${ }^{1}$, \\ Tatsuo OHTA ${ }^{2}$, Ei SAKAGUCHI ${ }^{3}$ and Takashi ICHIYANAGI ${ }^{4, *}$ \\ ${ }^{1}$ Bourbon Institutes of Health, Bourbon Corporation, 1-3-1 Ekimae, Kashiwazaki, \\ Niigata 945-8611, Japan \\ ${ }^{2}$ Department of Applied Life Sciences, Niigata University of Pharmacy and Applied Life Sciences, \\ Akiha-ku, Niigata 956-8603, Japan \\ ${ }^{3}$ Graduate School of Natural Science and Technology, Okayama University, 1-1-1 Tsushima-naka, \\ Okayama, Okayama 700-8530, Japan \\ ${ }^{4}$ Department of Environmental Science, Niigata Institute of Technology, 1719 Fujihashi, \\ Kashiwazaki, Niigata 945-1195, Japan
}

(Received November 5, 2014)

\begin{abstract}
Summary Boysenberry, a hybrid Rubus berry, is mainly cultivated in New Zealand. We previously reported that consumption of boysenberry juice (BBJ) exhibited anti-obesity effects in high-fat feeding rats. In this study, we focused on the suppressive effect of BBJ and its fraction on triglyceride absorption from the gastrointestinal tract. BBJ effectively inhibited pancreatic lipase activity in vitro, and was separated into four fractions ( $\mathrm{Fr} 1, \mathrm{Fr} 2, \mathrm{Fr} 3$ and Fr4) by HP-20 column chromatography. Among all the fractions, Fr3, the ellagic acid-rich fraction, showed the most potent inhibition against pancreatic lipase in vitro with $\mathrm{Fr} 2$, the anthocyanin-rich fraction, second. Authentic ellagic acid equivalent in $\mathrm{Fr} 3$ showed poor activity against pancreatic lipase. Then, each fraction was orally administered with corn oil to rats fitted with a jugular catheter to examine the effects of each fraction on plasma triglyceride levels. Both Fr2 and Fr3 effectively suppressed the plasma triglyceride level elevation at a dose of $1,000 \mathrm{mg} / \mathrm{kg}$ body weight. These findings demonstrated that BBJ contains chemical components which inhibit triglyceride absorption from the gastrointestinal tract.
\end{abstract}

Key Words boysenberry, anti-obesity effect, pancreatic lipase, polyphenol, lipid absorption

Recently, obesity caused by a lack of exercise and consumption of hyper-alimentation meals has become a problem worldwide (1). Obesity involving visceral fat accumulation induces hypertension and hyperlipidemia, leading to an increased risk of metabolic syndrome (2). Thus, prevention of obesity is an important strategy for avoiding metabolic syndrome (3). Some polyphenols, such as polymeric flavan-3-ols in oolong tea, exhibit anti-obesity effects to suppress lipid absorption from the small intestine (4). Green tea polyphenols, including epigallocatechin gallate, promote lipid metabolism (5).

Boysenberry is considered to be a hybrid Rubus berry bred between Rubus loganobaccus and Rubus baileyanus, and is mainly cultivated in New Zealand. This fruit is rich not only in nutrients such as iron, zinc, folic acid and vitamins (6), but also in some polyphenolic compounds such as anthocyanins, ellagic acid and ellagitannins $(7,8)$, as shown in Fig. 1. Recent studies have indicated that orally administered boysenberry juice (BBJ) shows positive biological effects such as reduction of oxidative stress $(9,10)$, prevention of liver injury (11) and anti-hypertension (12).

We previously reported that long-term feeding of BBJ

*To whom correspondence should be addressed.

E-mail: ichiyanagi@niit.ac.jp showed anti-obesity effects in rats served a high-fat diet (13) and significant suppression of postprandial plasma triglyceride (TG) levels were observed together with the lowering of hepatic fatty acid synthase (FAS) activity. Inhibition of TG absorption from gastrointestinal tract is thought to be an effective strategy to prevent obesity. Therefore, in this study, we focused on the suppressive effect of BBJ on triglyceride absorption from the gastrointestinal tract after a single oral administration of corn oil and attempted to determine the active components in BBJ by fractionation.

\section{MATERIALS AND METHODS}

Reagents. Ellagic acid and cholic acid were purchased from Wako Pure Chemical Industries, Ltd. (Osaka, Japan). 4-Methylumbelliferyl oleate (4MUO) and lipase from porcine pancreas were purchased from Sigma-Aldrich (Tokyo, Japan). A corn oil manufactured by Ajinomoto (Tokyo, Japan) was used for in vivo experiments. Cyanidin-3-O- $\beta$-D-glucoside chloride was purchased from Tokiwa Phytochemical Co., Ltd. (Chiba, Japan). Other reagents were purchased from Kanto Chemical Co., Inc. (Tokyo, Japan) without further purification. BBJ was obtained from Berryfruit Export NZ, Ltd. (Nelson, New Zealand) (Bx 65.1-65.2 ${ }^{\circ}$ ) together with other berry juices (blueberry, blackcurrant, red rasp- 


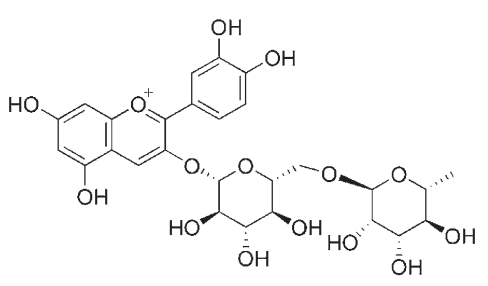

(1)

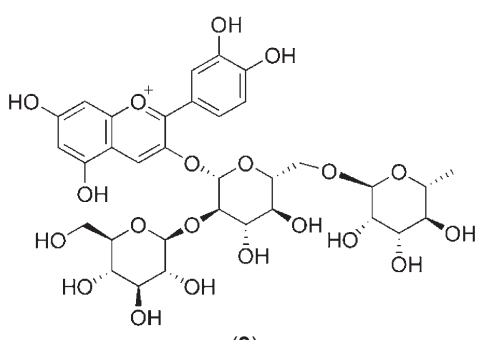

(3)

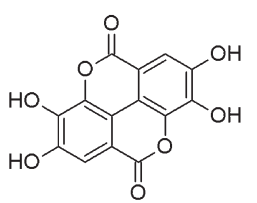

(5)<smiles>OC[C@H]1O[C@@H](Oc2cc3c(O)cc(O)cc3[o+]c2-c2ccc(O)c(O)c2)[C@H](O)[C@@H](O)[C@@H]1O</smiles>

(2)<smiles>OC[C@H]1O[C@@H](O[C@@H]2O[C@H](CO)[C@H](O)C(O)[C@H]2O)[C@H](Oc2cc3c(O)cc(O)cc3[o+]c2-c2ccc(O)c(O)c2)[C@@H](O)[C@@H]1O</smiles>

(4)

Fig. 1. Anthocyanins and ellagic acid in boysenberry. (1) cyanidin-3-[(6-rhamnosyl)glucoside], (2) cyanidin-3-glucoside, (3) cyanidin-3-[(2-glucosyl)-6-(rhamnosyl)glucoside], (4) cyanidin-3-[2-(glucosyl)glucoside], (5) ellagic acid.

berry, and grape) (Bx 65.1-65.2 ${ }^{\circ}$ ) for comparison. Each juice was stored at $-18^{\circ} \mathrm{C}$ until examination.

Fractionation of BBJ. BBJ was diluted with distilled water and loaded onto a column packed with Diaion ${ }^{\circledR}$ HP-20 resin (Mitsubishi Chemical Corporation, Tokyo, Japan). The column was washed with distilled water, and components of $\mathrm{BBJ}$ were subsequently recovered with ethanol. The water fraction (Fr1) was freeze-dried. The ethanol fraction was concentrated to remove the organic solvent in vacuo at $40^{\circ} \mathrm{C}$; then the remaining water fraction was removed and powdered by freezedrying. The ethanol fraction was resolved in distilled water and loaded onto the same column to achieve further separation. After washing the column with distilled water, two fractions (Fr2 and Fr3) were successively recovered with 50\% aqueous methanol and 100\% methanol. The remaining components in the column were recovered with acetone (Fr4). The organic solvent in each fraction was removed with the same procedures described above and was powdered by freeze-drying.

Inhibitory effects on pancreatic lipase. The lipaseinhibitory effects of $\mathrm{BBJ}$ were evaluated by the method of Kurihara et al. (14), with slight modifications. Briefly, $20-\mu \mathrm{L}$ aliquots of various juices were diluted with

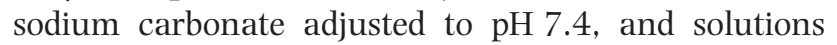
of the above fractions $(0-8 \mathrm{mg} / \mathrm{mL})$ were individually combined with $130 \mu \mathrm{L}$ of pancreatic lipase solution in $0.2 \mathrm{M}$ phosphate buffer $(0.6 \mathrm{mg} / \mathrm{mL})$ and incubated for $20 \mathrm{~min}$ at $37^{\circ} \mathrm{C}$. Next, $5 \mu \mathrm{L}$ of 4 MUO solution $(1 \mathrm{mM})$ was added to the mixtures. After incubation for $30 \mathrm{~min}$ at $37^{\circ} \mathrm{C}, 50 \mu \mathrm{L}$ of $5 \mathrm{M}$ acetic acid was added to stop the reaction. The fluorescence generated by $4 \mathrm{MUO}$ was measured using a fluorescence analyzer (Infinite M200; Tecan Japan Ltd., Kanagawa, Japan) with an excitation wavelength of $327 \mathrm{~nm}$ and an emission wavelength of $449 \mathrm{~nm}$. The inhibition of pancreatic lipase activity was calculated as a percentage against the control reaction. The 50\% inhibitory concentration $\left(\mathrm{IC}_{50}\right)$ value of each fraction was calculated from a mathematical formula obtained from linear approximation of curves as the logarithm of the concentration of each fraction versus the inhibitory activity.

Animal treatment. Male Wistar rats (7 wk of age) purchased from Japan SLC, Inc. (Shizuoka, Japan) were housed in an air-conditioned room $\left(22 \pm 2^{\circ} \mathrm{C}\right)$ with a 12-h/12-h light/dark cycle. The rats were allowed free access to tap water and a commercial diet (Rabo-MR Stock, Nihon Nosan Kogyo, Tokyo, Japan) for 6 d before the experiment.

After the 6-d conditioning period, 18 rats were cannulated with a polyethylene tube (PE-50) in the jugular vein under anesthesia with diethyl ether. Diethyl ether was inhaled for quick recovery of the rats from anesthesia before the administration study. The right jugular vein was isolated, and a small hole was made using scissors to insert the polyethylene tube. The tip of the tube was set on exactly the correct position of entrance of the right auricle of the rats. After insertion of the tube, the vein was occluded, and the tube penetrated the skin and was guided out from the back of the rat. After fast- 


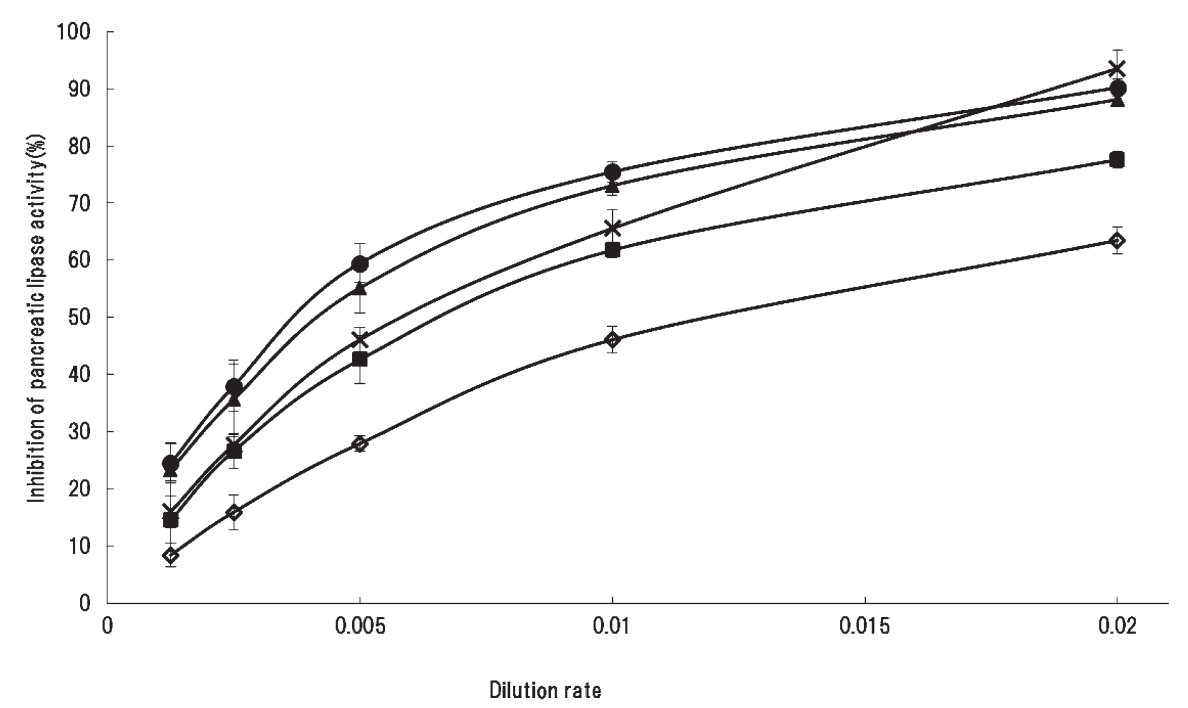

Fig. 2. Inhibitory effects of berry juices on pancreatic lipase. Liquid concentrates were from the same brix $\left(65.1-65.2^{\circ}\right)$. Data are expressed as percentages of the control levels. Values are means \pm SD $(n=4)$. Symbols: $\bullet$, BBJ; $\mathbf{\Delta}$, blueberry; $\times$, black currant; $\diamond$, red raspberry; $\mathbf{\square}$, grape.

ing for $20 \mathrm{~h}$, the rats were divided into three groups to make the mean body weight equal in each group. Emulsions were prepared by ultrasonic mixing of $5 \mathrm{~mL}$ of corn oil containing $200 \mathrm{mg}$ of cholic acid and $5 \mathrm{~mL}$ of each (none, Fr2 and Fr3) fraction dissolved in distilled water to adjust to an administration dose of 1,000 mg/ kg. Then, each emulsion was quickly administered to a different group of rats by direct stomach intubation (10 mL/kg body weight).

Blood samples $(0.2 \mathrm{~mL})$ were taken from each rat via the jugular catheter using a heparinized syringe at hourly intervals over a period of $10 \mathrm{~h}$, and centrifuged at $15,500 \times g$ for 10 min to obtain blood plasma. Then, concentrations of triglyceride (TG) in the blood plasma were quantified with a triglyceride test kit (Triglyceride E-test Wako; Wako Pure Chemical Industries). The area under the plasma concentration-time curve (AUC) was calculated from the time-dependent plasma TG profile over the 10 -h period following administration. The animals were maintained according to the Guidelines for Animal Experimentation of Okayama University, and the protocol was approved by the Ethical Committee at Okayama University.

Analysis of components in BBJ fraction. Qualitative and quantitative analyses of anthocyanins and ellagic acid were performed using a Shimadzu Prominence system (Shimadzu Corp., Kyoto, Japan) equipped with L-column 2 ODS $(2.1 \times 100 \mathrm{~mm}, 2 \mu \mathrm{m})$ (Chemicals Evaluation and Research Institute, Tokyo, Japan) at $40^{\circ} \mathrm{C}$. Compounds were eluted with a gradient system using 1\% aqueous formic acid (A) and 1\% formic acid in acetonitrile (B) as the elution solvent. The gradient system was as follows: $8 \% \mathrm{~B}$ at $0 \mathrm{~min}$ and a linear gradient of $18 \% \mathrm{~B}$ at $30 \mathrm{~min}$ at a flow rate of $0.2 \mathrm{~mL} / \mathrm{min}$. The injection volume of samples was $5 \mu \mathrm{L}$. The eluted components were monitored with a UV-Vis detector at $520 \mathrm{~nm}$ for anthocyanins and $350 \mathrm{~nm}$ for ellagic tannin. Anthocyanins and ellagic acid were quantified with commercially available authentic cyanidin 3-O- $\beta$ D-glucoside equivalent and ellagic acid. The eluent was further monitored with a triple quadrupole mass spectrometer, API 3200 (AB SCIEX, Framingham, MA), to obtain molecular and product ions. Total ion chromatograms were recorded over a mass range of $\mathrm{m} / \mathrm{z} 250$ to $\mathrm{m} / \mathrm{z} 800$ using a scan duration of 1 amu. Peaks giving $\mathrm{m} / \mathrm{z}$ values corresponding to possible anthocyanins in total ion chromatograms were further investigated by the product ion scan. Individual precursors and product ions were ionized via electrospray ionization (ESI) operated in the positive ion mode. Curtain gas and collision gas were applied as $\mathrm{N}_{2}$. The ion spray voltage and temperature were set at $5.5 \mathrm{kV}$ and $400^{\circ} \mathrm{C}$.

Statistics. The results of the in vitro assays are expressed as the mean \pm standard deviation (SD), while those of the in vivo assays are expressed as the mean \pm standard error (SE). The data were analyzed using a single factorial design for each sampling time, and examined for statistically significant differences by Tukey's multiple range test (Statcel 2 software; OMS Publishing, Saitama, Japan). A probability value of less than 0.05 was considered statistically significant.

\section{RESULTS}

Inhibitory effects of various berry juices on pancreatic lipase

BBJ showed the most potent inhibition among the examined juices at a dilution range from $1.25 \times 10^{-3}$ to $1 \times 10^{-2}$. Furthermore, BBJ, blueberry and blackcurrant juices showed similarly high inhibitory effects of more than $80 \%$ at a dilution of $2 \times 10^{-2}$, when the activity of the control was assumed to be $100 \%$ (Fig. 2).

Polyphenol components in BBJ fraction

BBJ, which showed the highest inhibition against pancreatic lipase, was separated into four fractions by HP-20 column chromatography. The recovery of each fraction was $55.3 \mathrm{~g}$ for Fr2, $20.1 \mathrm{~g}$ for Fr3 and $1.1 \mathrm{~g}$ for Fr4, when 1,500 g of BBJ was treated. Qualitative 
Table 1. Contents of anthocyanins and ellagic acid in Fr2, Fr3 and Fr4.

\begin{tabular}{|c|c|c|c|c|}
\hline \multirow{2}{*}{ Compounds } & \multirow{2}{*}{$\begin{array}{l}\text { Molecular mass } \\
\text { fragment }(\mathrm{m} / \mathrm{z})\end{array}$} & \multicolumn{3}{|c|}{ Content (mg/g) } \\
\hline & & Fr2 & Fr3 & Fr4 \\
\hline Cyanidin-3-[2-(glucosyl)glucoside] & $611 / 287$ & 56.3 & 1.08 & 29.3 \\
\hline Cyanidin-3-glucoside & $449 / 287$ & 21.1 & - & 11.9 \\
\hline Cyanidin-3-[2-(glucosyl)-6-(rhamnosyl)glucoside] & $757 / 287$ & 31.5 & - & 13.6 \\
\hline Cyanidin-3-[6-(rhamnosyl)glucoside] & 595 & 1.79 & 0.95 & - \\
\hline Total anthocyanins & & 111 & 2.03 & 54.8 \\
\hline Ellagic acid & & 1.21 & 108 & 0.21 \\
\hline
\end{tabular}

Pancreatic lipase inhibitory activity $\left(\mathrm{IC}_{50}\right)(\mathrm{mg} / \mathrm{mL})$

$\begin{array}{lll}0.33 & 0.17 & 0.36\end{array}$

and quantitative analyses demonstrated both Fr2 and Fr4 contained high amounts of anthocyanins. HPLCUV-MS/MS analysis showed that peaks 1, 2 and 3 had a molecular ion $\left(\mathrm{M}^{+}\right)$at $\mathrm{m} / \mathrm{z} 611,449$ and 757 , respectively, and a product ion $\left(\right.$ aglycone $^{+}$) at $\mathrm{m} / \mathrm{z} 287$. Peak 4 had a molecular ion $\left(\mathrm{M}^{+}\right)$at $\mathrm{m} / z$ at 595 . The total anthocyanin contents in the fractions were $111 \mathrm{mg} / \mathrm{g}$ for Fr2, $2.03 \mathrm{mg} / \mathrm{g}$ for Fr3 and $54.8 \mathrm{mg} / \mathrm{g}$ for Fr4 (Table 1). Similarly, contents of ellagic acid in each fraction were determined as 1.21, 108 and $0.21 \mathrm{mg} / \mathrm{g}$ for Fr2, Fr3 and Fr4, respectively.

Inhibitory effects of BBJ fractions on pancreatic lipase

Pancreatic lipase-inhibitory activity of BBJ fractions was evaluated in the same manner using the assay comparing the different berry juices. Fr1 did not inhibit pancreatic lipase $\left(\mathrm{IC}_{50},>0.8 \mathrm{mg} / \mathrm{mL}\right)$, while the other fractions inhibited pancreatic lipase in a concentrationdependent manner. The inhibition of lipase activity was as follows: $\mathrm{Fr} 3>\mathrm{Fr} 2=\mathrm{Fr} 4 \quad\left(\mathrm{IC}_{50}=0.17,0.33\right.$ and $0.36 \mathrm{mg} / \mathrm{mL}$, respectively) (Table 1). Because Fr3 contained the highest amount of ellagic acid, lipase-inhibitory activity also was examined for authentic ellagic acid. However, the authentic ellagic acid equivalent in Fr3 did not contribute to the inhibition of pancreatic lipase activity observed for Fr3 (data not shown).

Effects of BBJ fractions on plasma TG levels in rats

The plasma TG levels in the control group reached a maximum of $129.9 \pm 21.6 \mathrm{mg} / \mathrm{dL} 4 \mathrm{~h}$ after oral administration of the emulsion. The Fr2 and Fr3 groups both showed significant $(p<0.05)$ suppression of the elevation of plasma TG levels $3 \mathrm{~h}$ after the administration relative to the control group. Furthermore, both groups again showed significant $(p<0.05)$ suppression of plasma TG levels relative to the control group 6 to $7 \mathrm{~h}$ after oral administration (Fig. 3A). The AUC levels of TG after administration of the emulsion containing Fr2 and Fr 3 also were suppressed significantly $(p<0.05$ and $p<0.01$, respectively) compared with that of the control group (Fig. 3B).

\section{DISCUSSION}

Recent studies have demonstrated that several polyphenols such as flavanols (4), procyanidins (15) and hesperidin (16) showed potent lipase inhibition in vitro. Thus, consumption of food containing various polyphenols has attracted much attention to prevent obesity caused by a high-fat diet. We have previously reported BBJ significantly lowered plasma TG levels together with active FAS and cholesterol levels in the liver, leading to the suppression of elevations of total body weight and body fat in an obesity rat model induced by a high-fat diet including 30\% lard (13). These findings indicate that BBJ contains beneficial components associated with anti-obesity effects. BBJ contains high amounts of various polyphenolic components; therefore, in the present study, we focused on polyphenolic components in BBJ and obtained several fractions by column chromatography to evaluate lipase inhibition in vitro and suppression of plasma TG levels after a single oral administration of corn oil with $\mathrm{BBJ}$ fractions in rats. BBJ exerted the most potent inhibitory effects among all examined juices at a dilution range of $1.25 \times 10^{-3}$ to $1 \times 10^{-2}$. Therefore, BBJ was separated into several fractions by column chromatography for the prediction of active components. Both Fr2 and Fr3 showed strong lipase inhibition, while Fr1 recovered with distilled water including carbohydrates and organic acids showed poor activity.

Tandem mass spectrometry results revealed that peaks 1, 2 and 3 had a molecular ion $\left(\mathrm{M}^{+}\right)$at $m / z=611$, 449 and 757, respectively, and a product ion (aglycone $^{+}$) at $m / z=287$, which indicates carrying cyanidin as aglycone. The molecular ion $\left(\mathrm{M}^{+}\right)$of peak 4 was obtained at $\mathrm{m} / z=595$, although the product ion could not be observed. The anthocyanins in boysenberry were previously reported elsewhere (8). Taking the present results and previous reports into consideration, four anthocyanins in the BBJ fractions obtained in this study were determined as cyanidin-3-[2-(glucosyl)glucoside] for peak 1 , cyanidin-3-glucoside for peak 2 , cyanidin- 
(A)

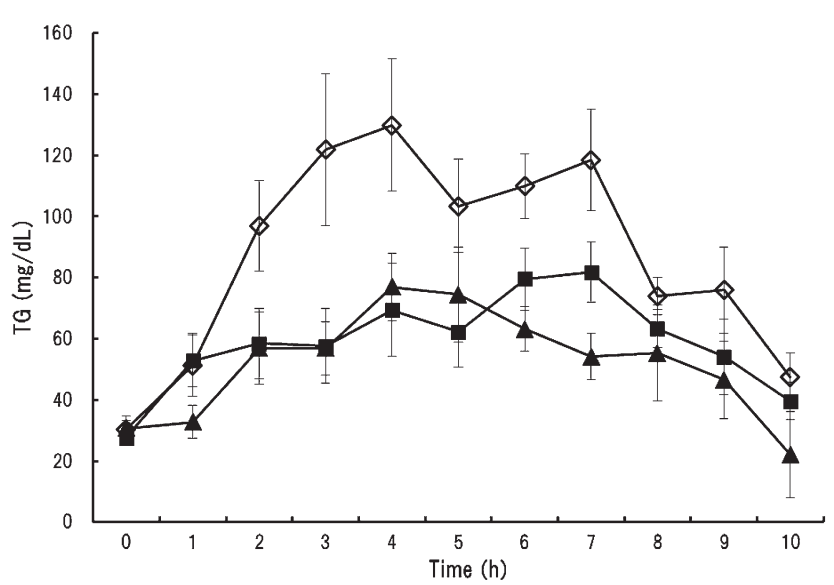

(B)

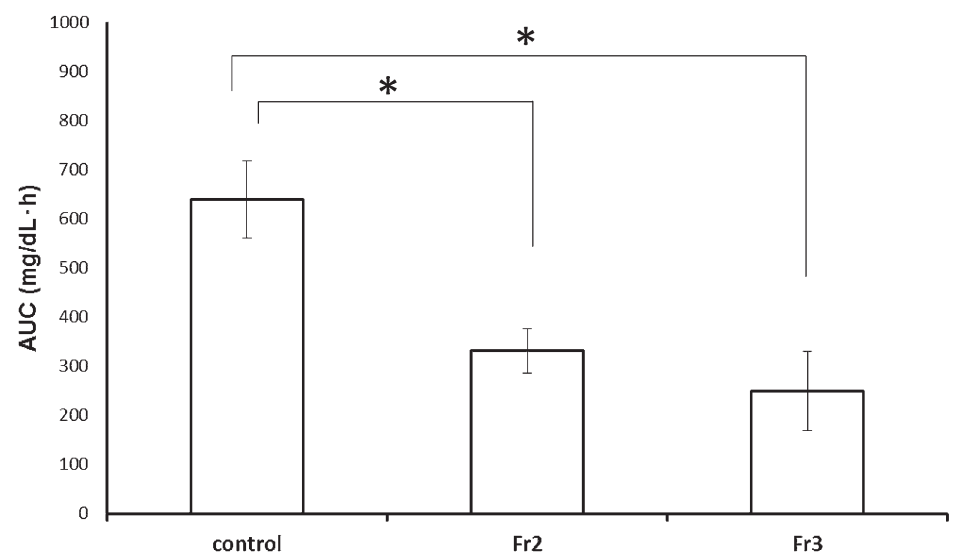

Fig. 3. Effects of Fr2 and Fr3 on the plasma TG levels after oral administration in rats. (A) Time-dependent changes in the plasma TG levels. Symbols: $\diamond$, control; $\mathbf{\square}$, Fr2; $\mathbf{\Delta}$, Fr3. (B) AUCs after a single oral dose of corn oil. Data are expressed as means \pm SE of $5-6$ rats. Values not sharing a common letter differ significantly at $p<0.05 .{ }^{*} p<0.05 ;{ }^{* *} p<0.01$ compared with the control group.

3-[(2-glucosyl)-6-(rhamnosyl)glucoside] for peak 3 and cyanidin-3-[(6-rhamnosyl)glucoside] for peak 4.

Fr3, the ellagic acid-rich fraction, expressed the most potent inhibitory activity among all examined fractions, although the authentic ellagic acid equivalent to $\mathrm{Fr} 3$ showed poor inhibitory effects on pancreatic lipase. This indicates the presence of other potent inhibitors of pancreatic lipase in Fr3. Furuuchi et al. (8) reported boysenberry contains quercetin glycosides and propelargonidin. Quercetins showed anti-obesity effects (17) based on inhibition of adipogenesis and apoptosis induction in 3T3-L1 cells (18), while inhibition by these polyphenols of lipid absorption from the gastrointestinal tract remains unclear. Therefore, these polyphenols may contribute to the lipase inhibitory activity of $\mathrm{Fr} 3$, although further studies are required to clarify the presence of these components in the fraction.

BBJ fractions (Fr2 and $\mathrm{Fr} 3$ ) were administered to jugular cannulated rats together with corn oil to confirm whether the suppression of plasma TG levels actually was expressed in vivo. Fr3 exhibited significant suppression of plasma TG levels after $3 \mathrm{~h}$ and 6 to $7 \mathrm{~h}$ after administration of corn oil in rats. The AUC level
$10 \mathrm{~h}$ after administration of Fr3 also was suppressed compared with control levels. The suppressive effect of Fr3 on the plasma TG elevation was stronger than that observed for Fr2. These results were coincident with those obtained in vitro, which was thought to be the strong inhibition of pancreatic lipase activity.

On the other hand, Fr2 also exhibited significant suppression of plasma TG levels after $3 \mathrm{~h}$ and 6 to $7 \mathrm{~h}$ after administration, although Fr2 showed weak lipase inhibition compared to that of Fr3. These results suggest Fr2 suppressed elevation of plasma TG levels by different pathways from pancreatic lipase inhibition. Inhibition of the constitution of the emulsion is another candidate mechanism for the Fr2-mediated suppression of the plasma TG levels. Numerous studies have demonstrated natural polyphenols inhibit the elevation of serum cholesterol levels in mammals (19-21). We have previously demonstrated that BBJ significantly decreased cholesterol levels in rat livers after long-term feeding of a highfat diet, similar to other polyphenols (13). Ikeda et al. (22) reported that tea catechins reduced the solubility of cholesterol in mixed micelles, leading to lower serum cholesterol levels. Thus, Fr2 could have the potential to 
inhibit lipid absorption from the gastrointestinal tract by inhibition of the constitution of the emulsion through binding of BBJ polyphenols to cholic acid, similar to the case of tea catechins. Further studies on the binding abilities of BBJ polyphenols to cholic acid should be examined.

Certain polyphenol components were thought to express their anti-obesity effect by modulating various factors related to lipid accumulation after being absorbed into adipose tissues. Tea catechins have been shown to increase acyl-CoA oxidase $(5,23)$ and (-)-epigallocatechin-3-gallate-inhibited lipid accumulation in 3T3-L1 cells (24). Anthocyanins in blueberry also ameliorated serum leptin levels (25) and authentic cyanidin 3-O-glucoside suppressed lipogenesis components at the mRNA level (26). In our previous work, BBJ significantly decreased active FAS activity in the liver (13) after longterm feeding of a high-fat diet containing BBJ in rats, although the same results were also obtained in rats consuming a normal diet containing BBJ. Therefore, we inferred that the polyphenols such as the anthocyanins contained in Fr2 may modulate adipocytes and enzymes promoting lipogenesis after absorption of these polyphenols, although further studies are required to clarify these points.

In conclusion, the anti-obesity effects of BBJ were, in part, the result of various polyphenolic components in BBJ through the inhibition of pancreatic lipase activity in the small intestine leading to the result of suppression of triglyceride absorption from the gastrointestinal tract.

\section{Acknowledgments}

The authors gratefully acknowledge Mr. Taichi Kazama for his advice with the HPLC-UV-MS/MS analysis.

\section{REFERENCES}

1) Nguyen DM, El-Serag HB. 2010. The epidemiology of obesity. Gastroenterol Clin North Am 39: 1-7.

2) Ritchie SA, Connell JMC. 2007. The link between abdominal obesity, metabolic syndrome and cardiovascular disease. Nutr Metab Cardiovasc Dis 17: 319-326.

3) Hosogai N, Fukuhara A, Oshima K, Miyata Y, Tanaka S, Segawa K, Furukawa S, Tochino Y, Komuro R, Matsuda M, Shimomura I. 2007. Adipose tissue hypoxia in obesity and its impact on adipocytokine dysregulation. Diabetes 56: 901-911.

4) Toyoda-Ono Y, Yoshimura M, Nakai M, Fukui Y, Asami S, Shibata H, Kiso Y, Ikeda I. 2007. Suppression of postprandial hypertriglyceridemia in rats and mice by oolong tea polymerized polyphenols. Biosci Biotechnol Biochem 71: 971-976.

5) Murase T, Nagasawa A, Suzuki J, Hase T, Tokimitsu I. 2002. Beneficial effects of tea catechins on diet-induced obesity: stimulation of lipid catabolism in the liver. Int $J$ Obes Relat Metab Disord 26: 1459-1464.

6) Lesperance L, Clark Z, Sivakumaran S, Sharp K. 2009. The Concise New Zealand Food Composition Tables, Edition No. 8, p 45. New Zealand Institute for Plant and Food Research, Palmerston North, Ministry of Health, Wellington, New Zealand.

7) Kool MM, Comeskey DJ, Cooney JM, McGhie TK. 2010.
Structural identification of the main ellagitannins of a boysenberry (Rubus loganbaccus $\times$ baileyanus Britt.) extract by LC-ESI-MS/MS, MALDI-TOF-MS and NMR spectroscopy. Food Chem 119: 1535-1543.

8) Furuuchi R, Yokoyama T, Watanabe Y, Hirayama M. 2011. Identification and quantification of short oligomeric proanthocyanidins and other polyphenols in boysenberry seeds and juice. J Agric Food Chem 59: 3738-3746.

9) Wada L, Ou B. 2002. Antioxidant activity and phenolic content of Oregon caneberries. J Agric Food Chem 50: 3495-3500.

10) Barnett LE, Broomfield AM, Hendriks WH, Hunt MB, McGhie, TK. 2007. The in vivo antioxidant action and the reduction of oxidative stress by boysenberry extract is dependent on base diet constituents in rats. J Med Food 10: $281-289$.

11) Igarashi K, Sugimoto E, Hatakeyama A, Molyneux J, Kubomura K. 2004. Preventive effects of dietary boysenberry anthocyanins on galactosamine-induced liver injury in rats. BioFactors 21: 259-261.

12) Furuuchi R, Sakai H, Hirokawa N, Watanabe Y, Yokoyama T, Hirayama M. 2012. Antihypertensive effect of boysenberry seed polyphenols on spontaneously hypertensive rats and identification of orally absorbable proanthocyanidins with vasorelaxant activity. Biosci Biotechnol Biochem 76: 1694-1701.

13) Noguchi A, Moriyama A, Mineo S, Fujisawa Y, Sugiyama M, Sakaguchi E. 2011. Effects of long-term boysenberry juice intake on lipid accumulation in rats. Nippon Eiyo Shokuryo Gakkaishi (J Jpn Soc Nutr Sci) 64: 27-33 (in Japanese).

14) Kurihara H, Asami S, Shibata H, Fukami H, Tanaka T. 2003. Hypolipemic effect of Cyclocarya paliurus (Batal) Iljinskaja in lipid-loaded mice. Biol Pharm Bull 26: 383-385.

15) Goncalves R, Mateus N, de Freitas V. 2010. Study of the interaction of pancreatic lipase with procyanidins by optical and enzymatic methods. J Agric Food Chem 58: 11901-11906.

16) Kawaguchi K, Mizuno T, Aida K, Uchino K. 1997. Hesperidin as an inhibitor of lipases from porcine pancreas and Pseudomonas. Biosci Biotechnol Biochem 61: 102104.

17) Ahn J, Lee H, Kim S, Park J, Ha T. 2008. The anti-obesity effect of quercetin is mediated by the AMPK and MAPK signaling pathways. Biochem Biophys Res Commun 373: 545-549.

18) Yang JY, Della-Fera MA, Rayalam S, Ambati S, Hartzell DL, Park HJ, Baile CA. 2008. Enhanced inhibition of adipogenesis and induction of apoptosis in 3T3-L1 adipocytes with combinations of resveratrol and quercetin. Life Sci 82: 1032-1039.

19) Yashiro T, Nanmoku M, Shimizu M, Inoue J, Sato R. 2012. Resveratrol increases the expression and activity of the low density lipoprotein receptor in hepatocytes by the proteolytic activation of the sterol regulatory element-binding proteins. Atherosclerosis 220: 369-374.

20) Salgado JM, Oliveira AGC, Mansi DN, Donado-Pestana CM, Bastos CR, Marcondes FK. 2010. The role of black rice (Oryza sativa L.) in the control of hypercholesterolemia in rats. J Med Food 13: 1355-1362.

21) Prior RL, Wu X, Gu L, Hager T, Hager A, Wilkes S, Howard L. 2009. Purified berry anthocyanins but not whole berries normalize lipid parameters in mice fed an obeso- 
genic high fat diet. Mol Nutr Food Res 53: 1406-1418.

22) Ikeda I, Imasato $Y$, Sasaki E, Nakayama M, Nagao H, Takeo T, Yayabe F, Sugano M. 1992. Tea catechins decrease micellar solubility and intestinal absorption of cholesterol in rats. Biochim Biophys Acta 1127: 141-146.

23) Dulloo AG, Duret C, Rohrer D, Girardier L, Mensi N, Fathi M, Chantre P, Vandermander J. 1999. Efficacy of a green tea extract rich in catechin polyphenols and caffeine in increasing 24-h energy expenditure and fat oxidation in humans. Am J Clin Nutr 70: 1040-1045.

24) Moon HS, Chung CS, Lee HG, Kim TG, Choi YJ, Cho CS.
2007. Inhibitory effect of (-)-epigallocatechin-3-gallate on lipid accumulation of 3T3-L1 cells. Obesity (Silver Spring) 15: 2571-2582.

25) Prior RL, Wilkes SE, Rogers TR, Khanal RC, Wu X, Howard LR. 2010. Purified blueberry anthocyanins and blueberry juice alter development of obesity in mice fed an obesogenic high-fat diet. I Agric Food Chem 58: 3970-3976.

26) Tsuda T, Horio F, Uchida K, Aoki H, Osawa T. 2003. Dietary cyanidin 3-O- $\beta$-D-glucoside-rich purple corn color prevents obesity and ameliorates hyperglycemia in mice. J Nutr 133: 2125-2130. 